12,18

\title{
К модельному описанию электронного спектра графеноподобных Янус-структур
}

\author{
() С.Ю. Давыдов \\ Физико-технический институт им. А.Ф. Иофрфе РАН, \\ Санкт-Петербург, Россия \\ E-mail: Sergei_Davydov@mail.ru \\ Поступила в Редакцию 1 октября 2021 г. \\ В окончательной редакции 1 октября 2021 г. \\ Принята к публикации 11 октября 2021 г.
}

\begin{abstract}
Предложена модель Янус-структуры $C-A B-D$ как соединения, образованного взаимодействующими (через атомы $A$ и $B$ ) димерами $A-C$ и $B-D$, где атомы $A$ и $B$ находятся в узлах двумерной гексагональной решетки, а атомы $C$ и $D$ расположены по разные стороны от плоскости этой решетки. В рамках теории сильной связи методом функций Грина получено общее уравнения для определения закона дисперсии. Подробно рассмотрены частные случаи соединений $C-A A-D$ и $A-A B-B$, для которых закон дисперсии получен в аналитическом виде и определено влияние внешней механической деформации на зонные характеристики. Обсуждается вопрос о намагниченности Янус-структур.
\end{abstract}

Ключевые слова: модель взаимодействующих димеров, электронный спектр, влияние деформации, магнитные состояния.

DOI: 10.21883/FTT.2022.02.51939.218

\section{1. Введение}

Двумерными Янус-соединениями называют структуры, верхняя и нижняя поверхности которых построены из различающихся атомов или молекул [1-3]. Исследования электронного спектра таких структур началось в 2009 г. с графона-графена, каждый второй атом углерода которого связан с атомом водорода, причем атомы водорода лежат по одну сторону от графенового листа (ту же структуру можно рассматривать как графан, у которого с одной из поверхностей удален водород) [4]. Практически одновременно возник интерес к структурам, в которых место атомов водорода занимают атомы галогенов, а место графена - графеноподобные соединения (GLC), такие как гексагональный нитрид бора (h-BN), силицен, германен и т.п. $[5,6]$. Большое внимание уделяется Янус-структурам на основе дихалькагенидов переходных металлов [7,8]. Особое место занимают исследования магнитных состояний в Янусструктурах $[4,9,10]$.

Насколько известно автору, все теоретические работы по данной тематике выполнены в рамках DFT (density functional theory). Здесь мы предложим простую модель графеноподобных Янус-соединений (J-GLC), позволяющую получить аналитические выражения для зонных характеристик (включая эффективные массы носителей) и оценить, как на значения этих характеристик влияет внешняя механическая деформация. Обсудим также вопрос о намагниченности J-GLC.

\section{2. Электронный спектр: общие соотношения}

Структурная модель гексагонального фрагмента J-GLC представлена на рисунке. В дальнейшем мы используем результаты работы [11], где рассматривалась симметричная графеноподобная структура, отличающаяся от структуры J-GLC тем, что по обе стороны от GLC-листа расположены одинаковые атомы $C$.

Как и в работе [11], начнем с набора двух различных асимметричных димеров, состоящих из атомов $A, C$ и $B, D$, связанных соответственно интегралами перехода $t_{a c}$ и $t_{b d}$. Построим из этих димеров гексагональную двумерную решетку, включив взаимодействие $t_{a b}$ между ближайшими атомами $A$ и $B$. Такую структуру будем обозначать как $C-A B-D$. Обобщая результаты рабо-

\section{Janus-GLC structure}

Top view

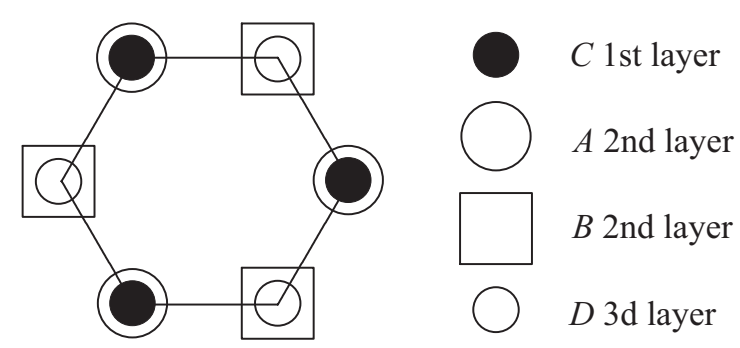

Гексагональный фрагмент структуры графеноподобного Януссоединения (J-GLC). 
ты [11], получим для J-GLC функции Грина вида

$$
\begin{gathered}
G^{A A(B B)}(\omega, \mathbf{k})=\frac{G^{A(B)}(\omega)}{1-t_{a b}^{2} G^{A}(\omega) G^{B}(\omega) f^{2}(\mathbf{k})}, \\
G^{A(B)}(\omega)=\frac{g^{A(B)}(\omega)}{1-t_{a(b) c(d)}^{2} g^{A(B)}(\omega) g^{C(D)}(\omega)},
\end{gathered}
$$

$$
f(\mathbf{k})=\sqrt{3+2 \cos \left(k_{x} a \sqrt{3}\right)+4 \cos \left(k_{x} a \sqrt{3} / 2\right) \cos \left(3 k_{y} a / 2\right)} .
$$

Здесь $\omega-$ энергетическая переменная, $g^{I}(\omega)=$ $=\left(\omega-\varepsilon_{i}+i 0^{+}\right)^{-1}$ - атомная функция Грина, где $\varepsilon_{i}-$ энергия уровня атома $i=a, b, c, d ; \mathbf{k}=\left(k_{x}, k_{y}, 0\right)-$ волновой вектор для движения электрона в плоскости $(x, y, 0), a-$ расстояние между ближайшими атомами $A$ и $B$. Электронный спектр системы находим из уравнения $\operatorname{Re} D(\omega, \mathbf{k})=0$, где

$$
\begin{aligned}
D(\omega, \mathbf{k})= & {\left[1-t_{a c}^{2} g^{A}(\omega) g^{C}(\omega)\right]\left[1-t_{b d}^{2} g^{B}(\omega) g^{D}(\omega)\right] } \\
& -t_{a b}^{2} g^{A}(\omega) g^{B}(\omega) f^{2}(\mathbf{k})
\end{aligned}
$$

откуда получаем уравнение

$$
\left(\Omega_{a} \Omega_{c}-t_{a c}^{2}\right)\left(\Omega_{b} \Omega_{d}-t_{b d}^{2}\right)-\Omega_{c} \Omega_{d} t_{a b}^{2} f^{2}(\mathbf{k})=0,
$$

где $\Omega_{i}=\omega-\varepsilon_{i}$. При $t_{a c}=t_{b d}=0$ имеем два локальных атомных уровня $\omega_{c, d}=\varepsilon_{c(d)}$ и две GLC-зоны $\omega_{a b}^{ \pm}(\mathbf{k})=\varepsilon_{a b} \pm \sqrt{\Delta_{a b}^{2}+t_{a b}^{2} f^{2}(\mathbf{k})}, \quad$ где $\quad \bar{\varepsilon}_{a b}=\left(\varepsilon_{a}+\varepsilon_{b}\right) / 2$ и $\Delta_{a b}=\left|\varepsilon_{a}-\varepsilon_{b}\right| / 2$. При $t_{a b}=0$ имеем четыре локальных уровня димеров $A-C$ и $B-D$ с энергиями $\bar{\omega}_{a c}^{ \pm}=\varepsilon_{a c} \pm R_{a c}$ и $\bar{\omega}_{b d}^{ \pm}=\varepsilon_{b d} \pm R_{b d}$, где $R_{i j}=\sqrt{\Delta_{i j}^{2}+t_{i j}^{2}}$, $\bar{\varepsilon}_{i j}=\left(\varepsilon_{i}+\varepsilon_{j}\right) / 2$ и $\Delta_{i j}=\left|\varepsilon_{i}-\varepsilon_{j}\right| / 2 \Delta_{i j}$. В общем случае решение уравнения (3) слишком громоздко и поэтому малоинформативно. Легко показать, однако, что при $\varepsilon_{a}=-\varepsilon_{b}, \varepsilon_{c}=-\varepsilon_{d}$ и $t_{a c}=t_{b d}$ уравнение (3) переходит в биквадратное уравнение. Поэтому ниже мы рассмотрим частные случаи и покажем, какие конкретные J-GLC этим случаям можно сопоставить.

\section{3. Частные случаи и соответствующие им J-GLC}

\section{1. Соединения $C-A A-D$}

Пусть $\varepsilon_{a}=\varepsilon_{b}=0, \varepsilon_{c}=-\varepsilon_{d}=e>0, t_{a c}=t_{b d}=t_{\perp}$, $t_{a b}=t$ (вариант 1). Тогда получаем решения уравнения (3) вида

$$
\begin{gathered}
E_{1 \pm}^{ \pm}(\mathbf{k})= \pm \sqrt{\left[B_{1}(\mathbf{k}) \pm R_{1}(\mathbf{k})\right] / 2} \\
R_{1}(\mathbf{k})=\sqrt{\left[e^{2}-t^{2} f^{2}(\mathbf{k})\right]^{2}+4 t_{\perp}^{2}\left[e^{2}+t^{2} f^{2}(\mathbf{k})\right]},
\end{gathered}
$$

где $B_{1}(\mathbf{k})=e^{2}+2 t_{\perp}^{2}+t^{2} f^{2}(\mathbf{k})$, верхние знаки у зон $E_{1 \pm}^{ \pm}(\mathbf{k})$ соответствуют знакам перед внешним корнем, нижние - перед внутренним корнем $R_{1}(\mathbf{k})$. В дальнейшем сосредоточим внимание на низкоэнергетических зонах $E_{1-}^{+}(\mathbf{k})=E_{C 1}(\mathbf{k})$ и $E_{1-}^{-}(\mathbf{k})=E_{V 1}(\mathbf{k})$, где индексы $C$ и $V$ относятся к зоне проводимости и валентной зоне.

Учитывая, что $f(\Gamma)=3, f(\mathrm{M})=1$ и $f(\mathrm{~K})=0$, где $\Gamma, \mathrm{M}$ и $\mathrm{K}$ - точки зоны Бриллюэна двумерной гексагональной решетки, имеем

$$
\begin{gathered}
B_{1 \Gamma}=e^{2}+2 t_{\perp}^{2}+9 t^{2}, \quad B_{1 \mathrm{M}}=e^{2}+2 t_{\perp}^{2}+t^{2}, \\
B_{1 \mathrm{~K}}=e^{2}+2 t_{\perp}^{2}, \quad R_{1 \Gamma}=\sqrt{\left(e^{2}-9 t^{2}\right)^{2}+4 t_{\perp}^{2}\left(e^{2}+9 t^{2}\right)}, \\
R_{1 \mathrm{M}}=\sqrt{\left(e^{2}-t^{2}\right)^{2}+4 t_{\perp}^{2}\left(e^{2}+t^{2}\right)}, \quad R_{1 \mathrm{~K}}=e \sqrt{e^{2}+4 t_{\perp}^{2}} .
\end{gathered}
$$

Щели между экстремумами низкоэнергетических зон в точках $\Gamma(\mathrm{M}, \mathrm{K})$ равны

$$
\Delta_{1 \Gamma(\mathrm{M}, \mathrm{K})}=\sqrt{2\left(B_{1 \Gamma(\mathrm{M}, \mathrm{K})}-R_{1 \Gamma(\mathrm{M}, \mathrm{K})}\right)} .
$$

Покажем теперь, что в рамках варианта 1 можно приближенно описать, например, соединение $\mathrm{Cl}$-графен $(\mathrm{Gr})-\mathrm{Br} . \quad \mathrm{B}$ принципе, можно подобрать соответствующие декорирующие атомные пары $C$ и $D$ и для других двумерных гексагональных одноэлементных структур типа силицена, германена и т.д., именуемых соединениями Xenens [12.13]. Здесь мы ограничимся асимметрично декорированным графеном.

Считая, что $\sigma$-связь атомов углерода $\left(\begin{array}{lll}A & \text { и } & B\end{array}\right)$ осуществляется $\left|s p^{2}\right\rangle$-орбиталями и принимая энергию $\varepsilon_{s p 2}(\mathrm{Gr})=\left(\varepsilon_{s}+2 \varepsilon_{p}\right) / 3=-11.82 \mathrm{eV}$ относительно вакуума (мы использовали таблицы атомных термов Хермана-Скиллмана [14]) за начало отсчета, получим для энергий $p$-уровней атомов хлора и брома значения -0.49 и $0.62 \mathrm{eV}$ соответственно. Для определения интегралов перехода $t$ и $t_{\perp}$ воспользуемся методом связывающих орбиталей Харрисона [15]. Тогда $t=V_{s p 2}=3.2\left(\hbar^{2} / m_{0} a^{2}\right)$, где $\hbar-$ приведенная постоянная Планка, $m_{0}$ - масса свободного электрона. В дальнейшем все длины связей будем приравнивать к сумме радиусов $r_{a}$ атомов [16,17], образующих эти связи. Тогда для $\mathrm{Gr}$ получаем длину связи $a=1.54 \AA$, что практически совпадает со значением $1.53 \AA$, полученным из численного расчета для графона $\mathrm{Gr}-\mathrm{C}$ [18]. Тогда получим $t \approx 10.5 \mathrm{eV}$. Связи атомов углерода с атомами галогенов считаем $\sigma$-связями двух $|p\rangle$-орбиталей, так что $t_{\perp}=V_{p p \sigma}=2.22\left(\hbar^{2} / m_{0} d^{2}\right)$, где $d-$ длина связи. Для атома углерода $r_{a}=0.77 \AA$ [16], для атомов $\mathrm{Cl}$ и $\mathrm{Br}$ значения $r_{a}$ (по Полингу) равны соответственно 0.99 и $1.14 \AA$ [17]. Отсюда получаем $t_{\perp}=5.46$ и $4.64 \mathrm{eV}$ для связей $\mathrm{C}-\mathrm{Cl}$ и $\mathrm{C}-\mathrm{Br}$ соответственно. Для дальнейших численных оценок положим $e=0.5 \mathrm{eV}, t=10 \mathrm{eV}$, $t_{\perp}=5 \mathrm{eV}$. Результаты расчетов сведены в таблицу, где приведены также оценки ширины зон разрешенных состояний $W_{C}=W_{V}=W=\left(\Delta_{\mathrm{K}}-\Delta_{\Gamma}\right) / 2$ и щели между высокоэнергетическими и низкоэнергетическими зонами $\Delta_{\mathrm{KK}}=E_{+}^{+}(\mathrm{K})-E_{-}^{+}(\mathrm{K})$.

Так как, $\Delta_{1 \Gamma}<\Delta_{1 \mathrm{~K}}$, ширина запрещенной зоны $E_{g 1}=\Delta_{1 \Gamma}$. В окрестности точки $\Gamma$ дисперсии зоны проводимости $E_{C}(\mathbf{k})=E_{-}^{+}(\mathbf{k})$ и валентной зоны 
Параметры модели и оценки зонных характеристик J-GLC (Bсе значения энергетических характеристик приведены в еV, деформационные характеристики нормированы на относительные деформации межатомных связей $\xi$ и $\xi$, отклики зонных характеристик на вариацию энергии е нормированы на $\psi$. Индексы 1 для $\mathrm{Cl}-\mathrm{Gr}-\mathrm{Br}$ и 2 для остальных соединений опущен.)

\begin{tabular}{|c|c|c|c|c|c|}
\hline J-GLC & $\mathrm{Cl}-\mathrm{Gr}-\mathrm{Br}$ & $\mathrm{B}-\mathrm{BN}-\mathrm{N}$ & $\mathrm{A} 1-\mathrm{AlP}-\mathrm{P}$ & $\mathrm{Ga}-\mathrm{GaAs}-\mathrm{As}$ & $\mathrm{In}-\mathrm{InSb}-\mathrm{Sb}$ \\
\hline$t$ & 10 & 10 & 5 & 4.4 & 3.3 \\
\hline$t_{\perp}$ & 5.0 & 10 & 5 & 4.4 & 3.3 \\
\hline$e$ & 0.5 & 3.0 & 2.2 & 1.3 & 1.5 \\
\hline $\begin{array}{c}\Delta_{\Gamma}=E_{g} \\
\delta_{2 D} \Delta_{\Gamma} / \xi \\
\delta_{z} \Delta_{\Gamma} / \xi \\
\delta_{e} \Delta_{\Gamma} / \psi\end{array}$ & $\begin{array}{c}1.89 \\
1.30 \\
-2.70 \\
0.25\end{array}$ & $\begin{array}{l}7.71 \\
4.65 \\
-51 \\
1.48\end{array}$ & $\begin{array}{l}4.63 \\
0.35 \\
-21 \\
0.80\end{array}$ & $\begin{array}{l}3.26 \\
1.17 \\
-24 \\
0.66\end{array}$ & $\begin{array}{l}3.07 \\
0.20 \\
-14 \\
0.93\end{array}$ \\
\hline $\begin{array}{c}\Delta_{\mathrm{M}} \\
\delta_{2 D} \Delta_{\mathrm{M}} / \xi \\
\delta_{z} \Delta_{\mathrm{M}} / \xi \\
\delta_{e} \Delta_{\mathrm{M}} / \psi\end{array}$ & $\begin{array}{c}4.22 \\
2.82 \\
-6.90 \\
0.09\end{array}$ & $\begin{array}{c}11.3 \\
3.22 \\
-34 \\
-0.92 \\
\end{array}$ & $\begin{array}{c}5.21 \\
0.52 \\
-18 \\
-0.75 \\
\end{array}$ & $\begin{array}{c}4.98 \\
1.45 \\
-15 \\
-0.39 \\
\end{array}$ & $\begin{array}{c}3.19 \\
0.12 \\
-13 \\
-0.50 \\
\end{array}$ \\
\hline $\begin{array}{c}\Delta_{\mathrm{K}} \\
\delta_{2 D} \Delta_{\mathrm{K}} / \xi \\
\delta_{z} \Delta_{\mathrm{K}} / \xi \\
\delta_{e} \Delta_{\mathrm{K}} / \psi\end{array}$ & $\begin{array}{c}9.51 \\
0 \\
-10 \\
-0.24 \\
\end{array}$ & $\begin{array}{c}17.8 \\
0 \\
-19 \\
-0.67 \\
\end{array}$ & $\begin{array}{c}8.68 \\
0 \\
-9 \\
-0.07 \\
\end{array}$ & $\begin{array}{c}7.83 \\
0 \\
-8 \\
-0.30 \\
\end{array}$ & $\begin{array}{c}5.73 \\
0 \\
-6 \\
-0.08 \\
\end{array}$ \\
\hline $\begin{array}{c}\Delta_{\mathrm{KK}} \\
\delta_{2 D} \Delta_{\mathrm{KK}} / \xi \\
\delta_{z} \Delta_{\mathrm{KK}} / \xi \\
\delta_{e} \Delta_{\mathrm{KK}} / \psi\end{array}$ & $\begin{array}{c}0.50 \\
0 \\
-4 \\
2 \\
\end{array}$ & $\begin{array}{c}2.90 \\
0 \\
-0.38 \\
10.8 \\
\end{array}$ & $\begin{array}{c}1.84 \\
0 \\
-0.56 \\
7.1 \\
\end{array}$ & $\begin{array}{c}1.26 \\
0 \\
-0.16 \\
4.71 \\
\end{array}$ & $\begin{array}{c}0.96 \\
0 \\
-0.63 \\
4.76 \\
\end{array}$ \\
\hline $\begin{array}{c}m_{e}^{*} \\
\delta_{2 D} m_{e}^{*} / m_{e}^{*} \xi \\
\delta_{z} m_{e}^{*} / m_{e}^{*} \xi \\
\delta_{e} m_{e}^{*} / m_{e}^{*} \psi\end{array}$ & $\begin{array}{c}5 \\
-4.9 \\
7 \\
0.13\end{array}$ & $\begin{array}{c}3 \\
-4.0 \\
30 \\
0.83\end{array}$ & $\begin{array}{c}8 \\
-4.5 \\
175 \\
7.27\end{array}$ & $\begin{array}{c}3 \\
-4.2 \\
60 \\
4.90\end{array}$ & $\begin{array}{c}11 \\
-4.5 \\
208 \\
1.13\end{array}$ \\
\hline$W$ & 3.81 & 2.90 & 2.03 & 2.20 & 1.31 \\
\hline
\end{tabular}

$E_{V}(\mathbf{k})=E_{-}^{-}(\mathbf{k})$ можно представить в виде

$$
E_{C(V)}(\mathbf{k})= \pm \frac{E_{g 1}}{2}+\frac{\hbar^{2} k^{2}}{2 m_{1 e(h)}} .
$$

где эффективные массы электронов $(e)$ и дырок $(h)$ равны

$$
m_{1 e}=-m_{1 h}=\frac{\hbar^{2} E_{g 1}}{9 t^{2} a^{2} C_{1 \Gamma}},
$$

$C_{1 \Gamma}=r_{1 \Gamma} / R_{1 \Gamma}-1, \quad r_{1 \Gamma}=9 t^{2}+2 t_{\perp}^{2}-e^{2} . \quad$ Значение безразмерной эффективной массы $m_{1 e}^{*}=m_{1 e} / m_{0}$ приведено в таблице. При $e^{2} \ll t_{\perp}^{2} \ll t^{2}$ получаем $C_{1 \Gamma} \approx 2 t_{\perp}^{4} / 81 t^{4} \ll 1$, точное значение $C_{1 \Gamma}=1.36 \cdot 10^{-3}$.

Теперь рассмотрим соединение $\mathrm{H}-\mathrm{Gr}-\mathrm{Cl}$, воспользовавшись таблицами атомных термов Фишер (см. [14], т. 2, Приложение 1). Тогда для атомов углерода (атомы $A$ и $B$ ) имеем $\varepsilon_{a}=\varepsilon_{b}=\left(\varepsilon_{s}+2 \varepsilon_{p}\right) / 3=-13.84 \mathrm{eV}$ относительно вакуума. Для атома водорода (атом $C$ ) имеем $\varepsilon_{c}=\varepsilon_{s}=-I=-13.60 \mathrm{eV}$, где $I-$ энергии ионизации. Для хлора имеем $\varepsilon_{d}=-13.78 \mathrm{eV}$. Положим, для простоты, $\varepsilon_{a}=\varepsilon_{b}=\varepsilon_{c}=\varepsilon_{d}=-13.7 \mathrm{eV}$ и примем эту энергию за начало отсчета. Далее, как показано выше, для связи $\mathrm{C}-\mathrm{Cl}$ имеем $t_{b d}=5.46 \mathrm{eV}$. По- лагая длину связи $\mathrm{C}-\mathrm{Cl}$ равной $1.30 \AA$, где за атомный радиус водорода мы приняли радиус Бора, получим $t_{a c}=V_{s p \sigma}=1.42\left(\hbar^{2} / m_{0} d^{2}\right)=6.40 \mathrm{eV}$. Положим $t_{\perp}=6 \mathrm{eV}$ и, как и выше, $t_{a b}=t=10 \mathrm{eV}$. Вместо уравнения (3) теперь имеем

$$
\omega^{4}-\left(2 t_{\perp}^{2}+t^{2} f^{2}(\mathbf{k})\right)+t_{\perp}^{4}=0,
$$

откуда получаем

$$
E_{ \pm}^{ \pm}(\mathbf{k})^{\prime}= \pm \sqrt{\left[B^{\prime}(\mathbf{k}) \pm R^{\prime}(\mathbf{k})\right] / 2}
$$

где

$$
B_{1}(\mathbf{k})=e^{2}=2 t_{\perp}^{2} f^{2}(\mathbf{k})
$$

И

$$
R^{\prime}(\mathbf{k})=t f(\mathbf{k}) \sqrt{4 t_{\perp}^{2}+t^{2} f^{2}(\mathbf{k})} .
$$

Таким образом, зоны $E_{ \pm}^{ \pm}(\mathbf{k})^{\prime}$ совпадают с зонами $E_{1 \pm}^{ \pm}(\mathbf{k})$ (4) для соединения $\mathrm{Cl}-\mathrm{Gr}-\mathrm{Br}$, где во всех выражениях для $B_{\Gamma, \mathrm{M}, \mathrm{K}}^{\prime}$ и $R_{\Gamma, \mathrm{M}, \mathrm{K}}^{\prime}$ нужно положить $e=0$. Так как для $\mathrm{Cl}-\mathrm{Gr}-\mathrm{Br} e \ll t_{\perp}, t$ (см. таблицу), а значения $t_{\perp}=5 \mathrm{eV}$ близко к $t_{\perp}=6 \mathrm{eV}$ для $\mathrm{H}-\mathrm{Gr}-\mathrm{Cl}$, будут близки и зонные характеристики двух этих соединений. Так, например, получаем $E_{g 1}^{\prime}=\Delta_{1 \Gamma}^{\prime}=2.31 \mathrm{eV}$ и $m_{1 e}^{\prime *}=3$. 
DFT-расчеты для соединений $\mathrm{H}-\mathrm{Gr}-D(D=\mathrm{F}, \mathrm{Cl}, \mathrm{Br})$ были выполнены в $[19,20]$. В этих работах рассматривались, однако, малые поверхностные концентрации атомов водорода и галогенов, образующих трехатомные островки вокруг гексагонов с обеих сторон графенового листа. В зависимости от конфигурации таких островков на поверхности графена меняются как положения запрещенных зон (точки $Г$ и К), так и их ширина (от 0.06 до $1.57 \mathrm{eV}$ ). Так, например, для соединения $\mathrm{H}-\mathrm{Gr}-\mathrm{Cl}$ для запрещенной зоны в точке Г было получено значение $E_{g}=1.57 \mathrm{eV}$. К сожалению, информация об эффективных массах носителей в $[19,20]$ отсутствует.

\section{2. Соединения $A-A B-B$}

Пуст теперь $\varepsilon_{a}=\varepsilon_{c}=e, \varepsilon_{b}=\varepsilon_{d}=-e, t_{a c}=t_{b d}=t_{\perp}$, $t_{a b}=t$ (вариант 2). Тогда из уравнения (3) получаем спектр вида

$$
\begin{gathered}
E_{2 \pm}^{ \pm}= \pm \sqrt{\left[B_{2}(\mathbf{k}) \pm R_{2}(\mathbf{k})\right] / 2} \\
R_{2}(\mathbf{k})=\sqrt{4 t_{\perp}^{2}\left[4 e^{2}+t^{2} f^{2}(\mathbf{k})\right]+t^{4} f^{4}(\mathbf{k})},
\end{gathered}
$$

где

$$
B_{2}(\mathbf{k})=2\left(e^{2}+t_{\perp}^{2}\right)=t^{2} f^{2}(\mathbf{k}) .
$$

Характерные зонные параметры равны:

$$
\begin{gathered}
B_{2 \Gamma}=2\left(e^{2}+t_{\perp}^{2}\right)+9 t^{2}, \quad B_{2 \mathrm{M}}=2\left(e^{2}+t_{\perp}^{2}\right)+t^{2}, \\
B_{2 \mathrm{~K}}=2\left(e^{2}+t_{\perp}^{2}\right), \quad R_{2 \Gamma}=\sqrt{4 t_{\perp}^{2}\left(4 e^{2}+9 t^{2}\right)+81 t^{4}} . \\
R_{2 \mathrm{M}}=\sqrt{4 t_{\perp}^{2}\left(4 e^{2}+t^{2}\right)+t^{4}}, \quad R_{2 \mathrm{~K}}=2 e t_{\perp},
\end{gathered}
$$

$\Delta_{2 \Gamma, \mathrm{M}}=2 \sqrt{\left(B_{2 \Gamma, \mathrm{M}}-R_{2 \Gamma, \mathrm{M}}\right) / 2}$ и $\Delta_{2 \mathrm{~K}}=2 \sqrt{e^{2}+t_{\perp}^{2}-e t_{\perp}}$. Легко показать, что $\Delta_{\Gamma}<\Delta_{\mathrm{K}}$, или $2 e t_{\perp}<R_{2 \Gamma}$, так что запрещенная зона формируется в точке $\Gamma$. Ширина зоны проводимости и валентной зоны $W=\left(\Delta_{\mathrm{K}}-\Delta_{\Gamma}\right) / 2$, а щель

$$
\Delta_{2 \mathrm{KK}}=\sqrt{e^{2}+t_{\perp}^{2}+e t_{\perp}}-\sqrt{e^{2}+t_{\perp}^{2}-e t} .
$$

Эффективные массы носителей имеют вид

$$
m_{2 e}^{*}=-m_{2 h}^{*}=\frac{\hbar^{2} E_{g 2}}{9 t^{2} a^{2} C_{2 \Gamma}},
$$

где $E_{g 2}=\Delta_{2 \Gamma}, C_{2 \Gamma}=r_{2 \Gamma} / R_{2 \Gamma}-1, r_{2 \Gamma}=9 t^{2}+2 t_{\perp}^{2}$.

Вариант 2 можно применить к J-GLC вида $A-A B-B$, где $A B$ представляют собой двумерные гексагональные соединения III-V, такие, например, как BN, AlP, GaAs и InSb $[11,21,22]$. Так как в рамках нашей схемы среднее значение длины связей $A-A$ и $B-B$ равно длине связи $A-B$, т.е. $d=a$, то $t_{\perp}=t$. Будем считать, что все атомы образуют $\sigma$-связи $\left|s p^{3}\right\rangle$-орбиталей с энергиями $\varepsilon_{s p 3}=\left(\varepsilon_{s}+3 \varepsilon_{p}\right) / 4$. Тогда $t=V_{s p 3}=3.22\left(\hbar^{2} / m_{0} a^{2}\right)[15]$. Принимая значение $\bar{\varepsilon}_{a b}=\left(\varepsilon_{a}+\varepsilon_{b}\right) / 2$ за начало отсчета энергии, положим $\varepsilon_{a}=-\varepsilon_{b}=e$. Результаты расчетов сведены в таблицу. Для сравнения приведем значение $E_{g}=2.55 \mathrm{eV}$, полученное для соединения $\mathrm{F}-\mathrm{BN}-\mathrm{H}$ в [9] - единственной известной нам работе по соединениям типа $C-A B-D$.

По результатам представленным в таблице, можно сформулировать следующие выводы: 1) соединения $\mathrm{Cl}-\mathrm{Gr}-\mathrm{Br}$ и $\mathrm{In}-\mathrm{InSb}-\mathrm{Sb}$ являются широкозонными полупроводниками, соединения $\mathrm{B}-\mathrm{BN}-\mathrm{N}, \mathrm{Al}-\mathrm{AlP}-\mathrm{P}$ и $\mathrm{Ga}-\mathrm{GaAs}-\mathrm{As}$ - изоляторами; 2) зоны $E_{C}(\mathbf{k})$ и $E_{V}(\mathbf{k})$ узки, мала и щель $\Delta_{\mathrm{KK}}$, отделяющая эти зоны от широких высокоэнергетических зон $E_{+}^{ \pm}(\mathbf{k})$; 3) в рассмотренных J-GLC эффективные массы носителей являются тяжелыми. В силу сделанных нами упрощений, все приведенные оценки зонных характеристик следует считать полуколичественными. Оценки эффективных масс являются лишь порядковыми, что связано с малостью параметра $C \sim 10^{-3}$, могущей приводить к существенным ошибкам в оценках. Малость $C$ возникает вследствие того, что при разложении выражения $E_{C}(\mathbf{k})=-E_{V}(\mathbf{k})$ по $(k a)^{2}$ вклады от $B(\mathbf{k})$ и $R(\mathbf{k})$ почти компенсируют друг друга, что приводит к слабой дисперсии (отсюда и узость зон) и большой эффективной массе.

Отметим, что оценки зонных параметров для графана $\mathrm{H}-\mathrm{Gr}-\mathrm{H}[23]$ и флюорографена $\mathrm{F}-\mathrm{Gr}-\mathrm{F}$ [24] (полученные в рамках практически той же модели, что и здесь) близки к результатам настоящей работы. Так, например, $E_{g} \approx 5.6$ и $3 \mathrm{eV}, m_{e}^{*}=-m_{h}^{*} \approx 2$ и 3 для графана и флюорографана соответственно. С другой стороны, согласно результатам работы [11], симметричные структуры $\mathrm{C}-\mathrm{BN}-\mathrm{C}, \mathrm{Si}-\mathrm{AlP}-\mathrm{Si}, \mathrm{Ge}-\mathrm{GaAs}-\mathrm{Ge}$ и $\mathrm{Sn}-\mathrm{InSb}-\mathrm{Sn}$ являются узкозонными полупроводниками $\left(E_{g} \approx 0.7 \mathrm{eV}\right.$ для $\mathrm{C}-\mathrm{BN}-\mathrm{C}$ и $\sim 0.3 \mathrm{eV}$ для остальных соединений), также узкими являются ширины зон $W_{C}=W_{V}$ и щели $\Delta_{\mathrm{KK}}$.

\section{4. Влияние деформации на электронный спектр}

Пусть гексагональная решетка, образованная атомами $A B$, подвергается всестороннему растяжению или сжатию (плоская деформация). Соответствующую относительную деформацию длины связи между ближайшими соседями обозначим $\xi=\delta a / a$. При такой деформации вид функции $f(\mathbf{k})$ не меняется, но энергетические характеристики зон претерпевают изменения. Пусть теперь под действием пары разнонаправленных вдоль оси $z$ сил осуществляется растяжение или сжатие связей $A-C$ и $B-D$. При этом деформации $\delta d_{a c}$ и $\delta d_{b d}$ длин связей должны удовлетворять равенству $k_{a c} \delta d_{a c}=k_{b d} \delta d_{b d}$, где $k_{a c}$ и $k_{b d}-$ силовые константы связей. В соответствии с $[14,15,25]$, центральная силовая константа $k=4 \alpha_{c}\left(1-2 \alpha_{p}\right) V_{2} / d^{2}$, где $V_{2}=t_{a c}, t_{b d}, \alpha_{c}=V_{2} / \sqrt{V_{2}^{2}+V_{3}^{2}}$ и $\alpha_{p}=V_{3} / \sqrt{V_{2}^{2}+V_{3}^{2}}-$ ковалентность и полярность связи, $V_{3}=\left|\varepsilon_{a(b)}-\varepsilon_{c(d)}\right| / 2$. 
Для рассматриваемых нами частных случаев $\left(d_{a c}=d_{b d}\right.$, $t_{a c}=t_{b d}=t_{\perp}$ и $\left.V_{2}=e / 2\right)$ получаем $\xi_{a c}=\xi_{b d}=\xi$.

\section{1. Соединения $C-A A-D$}

Перейдем теперь к оценкам для конкретных структур и начнем с соединений $C-A A-D$. Вводя для плоской деформации параметр $\delta_{2 D} L \equiv(\partial L / \partial a) \xi$, где $L-$ зонная характеристика, и учитывая, что $t_{a b} \propto a^{-2}$ и $t_{a c(b d)} \propto d_{a c(b d)}^{-2}$, получим:

$$
\begin{gathered}
\delta_{2 D} B_{1 \Gamma}=-36 t^{2} \xi, \delta_{2 D} B_{1 \mathrm{M}}=-4 t^{2} \xi, \delta_{2 D} B_{1 \mathrm{~K}}=0 \\
\delta_{2 D} R_{1 \Gamma}=\delta_{2 D} B_{1 \Gamma}\left(r_{1 \Gamma} / R_{1 \Gamma}\right), r_{1 \Gamma}=9 t^{2}+2 t_{\perp}^{2}-e^{2} \\
\delta_{2 D} R_{1 \mathrm{M}}=\delta_{2 D} B_{1 \mathrm{M}}\left(r_{1 \mathrm{M}} / R_{1 \mathrm{M}}\right), r_{1 \mathrm{M}}=t^{2}+2 t_{\perp}^{2}-e^{2} \\
\delta_{2 D} R_{1 \mathrm{~K}}=0 .
\end{gathered}
$$

Изменение параметров $\Delta_{1 \Gamma, \mathrm{M}, \mathrm{K}}$ равно:

$$
\delta_{2 D} \Delta_{1 \Gamma, \mathrm{M}, \mathrm{K}}=-\delta_{2 D} B_{1 \Gamma, \mathrm{M}, \mathrm{K}} C_{1 \Gamma, \mathrm{M}, \mathrm{K}} / 2 \Delta_{1 \Gamma, \mathrm{M}, \mathrm{K}},
$$

где $C_{1 \Gamma, \mathrm{M}, \mathrm{K}}=r_{1 \Gamma, \mathrm{M}, \mathrm{K}} / R_{1 \Gamma, \mathrm{M}, \mathrm{K}}-1$. Влияние плоской деформации на эффективные массы носителей дается выражением

$\delta_{2 D} m_{1 e(h)} / m_{1 e(h)}=2 \xi+\delta_{2 D} \Delta_{1 \Gamma} / \Delta_{1 \Gamma}+\frac{\delta_{2 D} B_{1 \Gamma}}{R_{1 \Gamma}}\left(1+\frac{r_{1 \Gamma}}{R_{1 \Gamma}}\right)$,

откуда получим $\delta_{2 D} m_{1 e(h)} / m_{1 e(h)} \approx-5.6 \xi$. Результаты расчета для соединений $\mathrm{Cl}-\mathrm{Gr}-\mathrm{Br}$ приведены в таблице. Те же по порядку величины результаты получим и для соединения $\mathrm{H}-\mathrm{Gr}-\mathrm{Cl}$. Таким образом, плоская деформация сравнительно слабо влияет на зонные параметры и эффективные массы носителей.

Перейдем к одноосной деформации, для чего определим параметр $\delta_{z} L \equiv(\partial L / \partial d) \xi$, где $\xi=\zeta_{a c}\left(1+k_{a c} / k_{b d}\right)$. Тогда получим

$$
\begin{aligned}
& \delta_{z} B_{1 \Gamma, \mathrm{M}, \mathrm{K}}=-8 t_{\perp}^{2} \zeta, \\
& \delta_{z} R_{1 \Gamma}=\delta_{z} B_{1 \Gamma}\left(\rho_{1 \Gamma} / R_{1 \Gamma}\right), \quad \rho_{1 \Gamma}=e^{2}+9 t^{2}, \\
& \delta_{z} R_{1 \mathrm{M}}=\delta_{z} B_{1 \mathrm{M}}\left(\rho_{1 \mathrm{M}} / R_{1 \mathrm{M}}\right), \quad \rho_{1 \mathrm{M}}=e^{2}+t^{2}, \\
& \delta_{z} R_{1 \mathrm{~K}}=\delta_{z} B_{1 \mathrm{~K}}\left(\rho_{1 \mathrm{~K}} / R_{1 \mathrm{~K}}\right), \quad \rho_{1 \mathrm{~K}}=e^{2} .
\end{aligned}
$$

Вместо (10) имеем

$$
\delta_{z} \Delta_{1 \Gamma, \mathrm{M}, \mathrm{K}}=\delta_{z} B_{1 \Gamma, \mathrm{M}, \mathrm{K}}\left(1-\rho_{1 \Gamma, \mathrm{M}, \mathrm{K}} / R_{1 \Gamma, \mathrm{M}, \mathrm{K}}\right) / 2 \Delta_{1 \Gamma, \mathrm{M}, \mathrm{K}} .
$$

откуда, с учетом неравенства $e^{2} \ll t_{\perp}^{2} \ll t^{2}$, находим

$$
\begin{aligned}
& \delta_{z} \Delta_{1 \Gamma} \approx-8 t_{\perp}^{4} \zeta / 9 t^{2} \Delta_{1 \Gamma}, \quad \delta_{z} \Delta_{1 \mathrm{M}} \approx-8 t_{\perp}^{4} \zeta / t^{2} \Delta_{1 \mathrm{M}}, \\
& \delta_{z} \Delta_{1 \mathrm{~K}} \approx-4 t_{\perp}^{4} \zeta / \Delta_{1 \mathrm{~K}}, \quad \delta_{z} \Delta_{1 \mathrm{KK}}=0 .
\end{aligned}
$$

Таким образом, $\left|\delta_{z} \Delta_{1 \Gamma, \mathrm{M}, \mathrm{K}}\right| \gg\left|\delta_{2 D} \Delta_{1 \Gamma, \mathrm{M}, \mathrm{K}}\right|$. Для эффективных масс получаем

$$
\delta_{z} m_{1 e(h)} / m_{1 e(h)}=\delta_{z} \Delta_{1 \Gamma} / \Delta_{1 \Gamma}-\frac{\delta_{2 D} B_{1 \Gamma}}{C_{1 \Gamma} R_{1 \Gamma}}\left(1-\frac{r_{1 \Gamma} \rho_{1 \Gamma}}{R_{1 \Gamma}^{2}}\right) .
$$

Результаты численных оценок для соединения $\mathrm{Cl}-\mathrm{Gr}-\mathrm{Br}$ приведены в таблице. Того же порядка оценки отвечают соединению $\mathrm{H}-\mathrm{Gr}-\mathrm{Cl}$. Отметим, вопервых, что деформации $\delta_{2 D} \Delta_{\Gamma, \mathrm{M}}>0\left(C_{1 \Gamma, \mathrm{M}, \mathrm{K}}>0\right)$ и растут при переходе от $\Gamma$ к $\mathrm{M}, \delta_{2 D} \Delta_{\mathrm{K}}=0$; деформации $\delta_{z} \Delta_{1 \Gamma, \mathrm{M}, \mathrm{K}}<0\left(\rho_{1 \Gamma, \mathrm{M}, \mathrm{K}} / R_{1 \Gamma, \mathrm{M}, \mathrm{K}}<1\right)$ и также растут по модулю при переходе от $Г$ к К. По порядку величины все деформационные характеристики лежат в диапазоне от 0 до $10 \xi(\xi) \mathrm{eV}$. Значение $\delta_{2 D} m_{1 e(h)} / m_{1 e(h)}=-4.9 \xi$, так как третье слагаемое в формуле (11) преобладает; $\delta_{z} m_{1 e(h)} / m_{1 e(h)}=7 \xi$, так как $r_{1 \Gamma} \rho_{1 \Gamma} / R_{1 \Gamma}<1$. Подчеркнем, что одноосная деформация сильнее изменяет характеристики электронного спектра, чем деформация плоская.

\section{2. Соединения $\boldsymbol{A}-\boldsymbol{A B}-\boldsymbol{B}$}

В случае плоской деформации легко показать, что $\delta_{2 D} B_{1 \Gamma, \mathrm{M}, \mathrm{K}}=\delta_{2 D} B_{1 \Gamma, \mathrm{M}, \mathrm{K}}$, а значения $\delta_{2 D} R_{2 \Gamma, \mathrm{M}, \mathrm{K}}$ даются формулами (9) с соответствующей заменой индекса 1 на 2, где $r_{2 \Gamma}=9 t^{2}+2 t_{\perp}^{2}, \quad r_{2 \mathrm{M}}=t^{2}+2 t_{\perp}^{2}$ $\left(r_{1 \Gamma, \mathrm{M}}\right.$ переходят в $r_{2 \Gamma, \mathrm{M}}$ при $\left.e=0\right)$. Для $\delta_{2 D} m_{2 e(h)}$ справедлива формула (10) с заменой индексов 1 на индексы 2. В случае одноосной деформации имеем $\delta_{z} B_{2 \Gamma, \mathrm{M}, \mathrm{K}}=-8 t_{\perp}^{2} e^{2}, \delta_{z} R_{2 \Gamma, \mathrm{M}}=\delta_{z} B_{2 \Gamma, \mathrm{M}}\left(\rho_{2 \Gamma, \mathrm{M}} / R_{2 \Gamma, \mathrm{M}}\right)$, $\rho_{2 \Gamma, \mathrm{M}}=e^{2}, \delta_{z} R_{2 \mathrm{~K}}=-4 t_{\perp} e$. Выражения для $\delta_{z} \Delta_{2 \Gamma, \mathrm{M}, \mathrm{K}}$ и $\delta_{z} m_{2 e}(h)$ получаются из выражений (13) и (14) заменой индексов 1 на индексы 2. Результаты численных оценок для двумерных гексагональных соединений III-V сведены в таблицу. Из таблицы, во-первых, следует, что реакция зонных параметров этих соединений на плоскую деформацию практически такая же, что и у соединений $\mathrm{Cl}-\mathrm{Gr}-\mathrm{Br}$ и $\mathrm{H}-\mathrm{Gr}-\mathrm{Cl}$. Что же касается отклика структур $A-A B-B$ на одноосную деформацию, то для рассмотренных здесь характеристик, соответствующих точкам $Г$ и М зоны Бриллюэна, этот отклик значительно выше (в разы и десятки раз), чем для соединений $\mathrm{Cl}-\mathrm{Gr}-\mathrm{Br}$ и $\mathrm{H}-\mathrm{Gr}-\mathrm{Cl}$. Причина такого расхождения состоит в том, что для $A-A B-B$ отношение $\rho_{2 \Gamma, \mathrm{M}} / R_{2 \Gamma, \mathrm{M}} \ll 1$ и $\left(1-r_{2 \Gamma, \mathrm{M}} \rho_{2 \Gamma, \mathrm{M}} / R_{2 \Gamma, \mathrm{M}}^{2}\right) \sim 1$, а для $\mathrm{Cl}-\mathrm{Gr}-\mathrm{Br}$ и $\mathrm{H}-\mathrm{Gr}-\mathrm{Cl}$ имеем $\rho_{2 \Gamma, \mathrm{M}} / R_{2 \Gamma, \mathrm{M}} \sim 1$ и $\left(1-r_{2 \Gamma, \mathrm{M}} \rho_{2 \Gamma, \mathrm{M}} / R_{2 \Gamma, \mathrm{M}}^{2}\right) \ll 1$. При этом для всех структур $C_{1 \Gamma} \sim C_{2 \Gamma} \ll 1$.

Итак, в этом разделе мы рассмотрели реакцию электронного спектра J-GLC на деформацию длин связей $a$ и $d$. Полученные результаты можно, однако, трактовать и как устойчивость модели к выбору параметров $a$ и $d$. В Приложении рассмотрен отклик системы на вариацию энергии $е$. Отметим, что длины и энергии связей являются единственными параметрами использованного нами здесь метода связывающих орбиталей Харрисона [14,15].

\section{5. О магнитных состояниях в J-GLC}

Вопрос о намагниченности J-GLC был поднят в теоретических работах $[4.10,26]$, где рассматривался графон 
$\mathrm{Gr}-\mathrm{H}$. Было показано, что свободный от $\mathrm{H}$ атом углерода обладает большим спиновым моментом $\left(\sim 1 \mu_{\mathrm{B}}\right.$, где $\mu_{\mathrm{B}}$ - магнетон Бора). Аналогичные результаты получены в [9] для соединения $\mathrm{H}-\mathrm{BN}$ и $\mathrm{F}-\mathrm{BN}$ : свободные атомы азота обладает магнитным моментом $\sim 0.75$ и $1 \mu_{\mathrm{B}}$ соответственно.

Для описания спиновых состояний в приближении Хартри-Фока в рамках предложенной нами модели J-GLC нужно, во-первых, приписать спиновый индекс $\sigma=\uparrow, \downarrow$ всем функциям Грина, входящим в выражение (1), и вместо энергии $\varepsilon_{i}(i=a, b, c, d)$ ввести энергию $\varepsilon_{i \sigma}=\varepsilon_{i}+U_{i} n_{i-\sigma}$, где $U_{i}-$ кулоновское отталкивание электронов с противоположными спинами на атоме $I=A, B, C, D, n_{i \sigma}$ - число заполнения уровня $\varepsilon_{i \sigma}[27,28]$. Во-вторых, оставляя в качестве декорирующих атомов только атомы С, следует в выражении (1) заменить функцию Грина $G_{\sigma}^{B}(\omega)$ димера $B-D$ на функцию Грина $g_{\sigma}^{B}(\omega)$ атома $B$. Тогда вместо (3) получим систему двух уравнений (для $\sigma=\uparrow, \downarrow)$ вида

$$
\Omega_{a \sigma} \Omega_{b \sigma} \Omega_{c \sigma}-\Omega_{b \sigma} t_{a c}^{2}-\Omega_{c \sigma} t_{a b}^{2} f^{2}(\mathbf{k})=0,
$$

где $\Omega_{i \sigma}=\omega-\varepsilon_{i \sigma}$. То же уравнение можно получить из (3), положив $t_{b d}=0$ и отбросив решение $\Omega_{d}=0$. В общем виде самосогласованное решение системы уравнений (15) является достаточно трудоемкой проблемой.

Рассмотрим, например, графон. Учитывая, что длины связей $\mathrm{C}-\mathrm{C}$ и $\mathrm{C}-\mathrm{H}$, равных соответственно 1.50 и $1.16 \AA \quad[9,18]$, имеем $t_{a b}=t \approx 11 \mathrm{eV}$ и $t_{a b}=V_{s p \sigma} \equiv t \approx 8 \mathrm{eV}$ [15]. Для атомов углерода $\varepsilon_{a}=\varepsilon_{b}=\left(\varepsilon_{s}+2 \varepsilon_{p}\right) / 3=-13.84 \mathrm{eV} \quad$ относительно вакуума, где мы использовали таблицы атомных термов Манна [29], содержащие также значение $U_{a}=U_{b}=11.76 \mathrm{eV}$ (отметим, что значения энергий $\varepsilon_{s}$ и $\varepsilon_{p}$ таблиц Манна практически совпадают со значениями таблиц Фишер). Для атома водорода $\varepsilon_{c}=\varepsilon_{s}=-I=-13.60 \mathrm{eV}, U_{c}=I-A=12.85 \mathrm{eV}$, где $I$ и $A-$ энергии ионизации и сродства к электрону [16]. Положим $\varepsilon_{a}=\varepsilon_{b}=\varepsilon_{c}=-13.7 \mathrm{eV}, \quad U_{a}=U_{b}=U_{c} \equiv U$ $=12.3 \mathrm{eV}$. Вводя локализованные на атоме $I$ спиновые моменты $m_{i}=n_{i \uparrow}-n_{i \downarrow}$ и полагая $\varepsilon_{a}+U / 2=\varepsilon_{b}+U / 2$ $=\varepsilon_{c}+U / 2=0, \quad$ получим $\quad \varepsilon_{i \uparrow}=-U\left(Z_{i}+m_{i}\right) / 2 \quad$ и $\varepsilon_{i \downarrow}=-U\left(Z_{i}-m_{i}\right) / 2, \quad$ где $Z_{i}=1-n_{i} \quad$ - заряд $i$-го атома. Учтем два дополнительных обстоятельства: 1) вследствие закона сохранения заряда имеем $\left.\sum_{i} Z_{i}=0 ; 2\right)$ так как в системе на элементарную ячейку приходится лишь один нескомпенсированный спин, локализованный, в основном, на неспаренной $\left|p_{z}\right\rangle$-орбитали атома углерода, получаем $\sum_{i} m_{i}=1$. Отсюда следует, что $\sum_{i} \varepsilon_{i}=U$.

Без учета кулоновского взаимодействия $(U=0)$ из уравнения (3) получаем симметричные зоны вида $E_{0}^{0}(\mathbf{k})=0$ и $E_{ \pm}^{0}(\mathbf{k})= \pm \sqrt{t_{\perp}^{2}+4 t^{2} f^{2}(\mathbf{k})}$. При $U \neq 0$ симметрия нарушается за счет межатомного перехода электронов и наличия спиновых моментов. Легко видеть, что даже при сделанных нами значительных упрощениях самосогласованная система уравнений (15) не имеет аналитического решения и следует обращаться к машинным расчетам.

\section{6. Заключение}

Итак, в настоящей работе мы предложили модель Янус-структуры и рассмотрели ряд случаев, для которых эта модель дает простые аналитические решения. Отметим, что такая модель удобна для экспресс-оценок зонных параметров (щелей в спектре, эффективных масс), отклика на внешнее возмущение (в нашем случае, механическую деформацию) и, в случае рассмотрения достаточно представительного набора изучаемых соединений, выявления характера изменений той или иной физической характеристики. При этом мы не использовали какие-либо подгоночные параметры, определяя энергии состояний по таблицам атомных термов [14,29], межатомные расстояния - по сумме атомных радиусов $[16,17]$ и интегралы перехода - по схеме Харрисона $[14,15]$. В принципе, не составило бы большого труда, воспользовавшись подходами работ по графану [23], флюорографену [24] и графеноподобным соединениям $C-h-A B-C[11]$, рассмотреть здесь емкостные характеристики Янус-структур, а также и вопрос о дефектах в этих структурах. Мы, однако, отложили эти исследования до появления соответствующих экспериментальных данных или, хотя бы, расчетов из первых принципов.

\section{Конфликт интересов}

Автор заявляет об отсутствии конфликта интересов.

\section{ПРИЛОЖЕНИЕ}

Рассмотрим реакцию электронного спектра на вариации энергии $e$, определив реакцию зонной характеристики $L$ на относительное изменение $\psi=\delta e / e$ как $\delta_{e} L=(\partial L / \partial e) \psi$.

\section{Соединения $C-A A-D$.}

В соответствии с выражениями для $B_{1 \Gamma, \mathrm{M}, \mathrm{K}}$ и $R_{1 \Gamma \mathrm{M}, \mathrm{K}}$ имеем

$$
\begin{gathered}
\delta_{e} B_{1 \Gamma, \mathrm{M}, \mathrm{K}}=2 e^{2} \psi, \quad \delta_{e} R_{1 \Gamma}=-2 e^{2}\left(9 t^{2}-4 t_{\perp}^{2}-e^{2}\right) \psi / R_{1 \Gamma}, \\
\delta_{e} R_{1 \mathrm{M}}=-2 e^{2}\left(t^{2}-4 t_{\perp}^{2}-e^{2}\right) \psi / R_{1 \mathrm{M}}, \\
\delta_{e} R_{1 \mathrm{~K}}=R_{1 \mathrm{~K}}\left(1+e^{4} / R_{1 \Gamma}^{2}\right) \psi
\end{gathered}
$$

Для реакции щелей $\Delta_{1 \Gamma, \mathrm{M}, \mathrm{K}}, \Delta_{1 \mathrm{KK}}$ и эффективных масс носителей $m_{1 e}=-m_{1 h}$ на относительное изменение энергии $e$ получаем

$$
\delta_{e} \Delta_{1 \Gamma, \mathrm{M}, \mathrm{K}}=\left(\delta_{e} B_{1 \Gamma, \mathrm{M}, \mathrm{K}}-\delta_{e} R_{1 \Gamma, \mathrm{M}, \mathrm{K}}\right) / 2 \Delta_{1 \Gamma, \mathrm{M}, \mathrm{K}},
$$




$$
\begin{aligned}
\delta_{e} \Delta_{1 \mathrm{KK}}= & \left(\delta_{e} B_{1 \mathrm{~K}}+\delta_{e} R_{1 \mathrm{~K}}\right) / \sqrt{\left(B_{1 \mathrm{~K}}+R_{1 \mathrm{~K}}\right) / 2} \\
& -\left(\delta_{e} B_{1 \mathrm{~K}}-\delta_{e} R_{1 \mathrm{~K}}\right) / \sqrt{\left(B_{1 \mathrm{~K}}-R_{1 \mathrm{~K}}\right) / 2} . \\
\delta_{e} m_{1 e}= & -\delta_{e} m_{1 h}=m_{1 e}\left(\delta_{e} \Delta_{1 \Gamma} / \Delta_{1 \Gamma}-\delta_{e} C_{1 \Gamma} / C_{1 \Gamma}\right), \\
\delta_{e} C_{1 \Gamma}= & -\frac{2 e^{2} \psi}{R_{1 \Gamma}}\left(1-\frac{r_{1 \Gamma}\left(9 t^{2}-4 t_{\perp}^{2}-e^{2}\right)}{R_{1 \Gamma}^{2}}\right) .
\end{aligned}
$$

Результаты численных оценок для соединения $\mathrm{Cl}-\mathrm{Gr}-\mathrm{Br}$ приведены в таблице. Для соединения $\mathrm{H}-\mathrm{Gr}-\mathrm{Cl}$, для которого $e=0$, и, как следствие, $\delta_{e} B_{1 \Gamma, \mathrm{M}, \mathrm{K}}=\delta_{e} R_{1 \Gamma, \mathrm{M}, \mathrm{K}}=0, \quad$ получаем $\quad \delta_{e} \Delta_{1, \mathrm{M}, \mathrm{K}}=\delta_{e} \Delta_{\mathrm{KK}}$ $=\delta_{e} m_{1 e(h)}=0$.

\section{Соединения $\boldsymbol{A}-\boldsymbol{A B}-\boldsymbol{B}$.}

В соответствии с выражениями для $B_{2 \Gamma, \mathrm{M}, \mathrm{K}}$ и $R_{2 \Gamma, \mathrm{M}, \mathrm{K}}$ имеем

$$
\begin{gathered}
\delta_{e} B_{2 \Gamma, \mathrm{M}, \mathrm{K}}=4 e^{2} \psi, \\
\delta_{e} R_{2 \Gamma, \mathrm{M}}=16 e^{2} t_{\perp}^{2} \psi / R_{2 \Gamma, \mathrm{M}}, \quad \delta_{e} R_{2 \mathrm{~K}}=R_{2 \mathrm{~K}} \psi .
\end{gathered}
$$

Реакции щелей $\Delta_{2 \Gamma, \mathrm{M}, \mathrm{K}}, \Delta_{2 \mathrm{KK}}$ даются формулами (П1) и (П2) с заменой индексов 1 на индексы 2. Для эффективных масс получаем

$$
\begin{gathered}
\delta_{e} m_{2 e}=-\delta_{e} m_{2 h}=m_{1 e}\left(\delta_{e} \Delta_{2 \Gamma} / \Delta_{1 \Gamma}-\delta_{e} C_{2 \Gamma} / C_{2 \Gamma}\right), \\
\delta_{e} C_{2 \Gamma}=-r_{2 \Gamma} \delta_{e} R_{2 \Gamma} / R_{2 \Gamma}^{2} .
\end{gathered}
$$

Численные результаты приведены в таблице, из которой следует, что изменения почти всех рассмотренных характеристик по модулю не превышают $\psi$. Исключениями являются значения $\delta_{e} \Delta_{2 \Gamma}$ для $\mathrm{B}-\mathrm{BN}-\mathrm{N}$ и $\delta_{e} m_{e} / m_{e}$ для $\mathrm{Al}-\mathrm{AlP}-\mathrm{P}, \mathrm{Ga}-\mathrm{GaAs}-\mathrm{As}$ и $\mathrm{In}-\mathrm{InSb}-\mathrm{Sb}$. Порядок величины $\psi$ логично оценить по разнице энергий атомных уровней $\varepsilon_{s}$ и $\varepsilon_{p}$, приведенных в таблицах Манна (M) [15] и Хермана-Скиллмана (HS) [14]. Оценки $\psi_{s, p}=2\left[\varepsilon_{s, p}(\mathrm{M})-\varepsilon_{s, p}(\mathrm{HS})\right]$ показывают, что максимальные значения $\left(\psi_{z}\right)_{\max }=0.17$ имеет место для атома хлора и $\left(\psi_{p}\right)_{\max }=0.24$ для атома брома. Средние значения для всех атомов, составляющих рассмотренные нами J-GLC, равны $\bar{\psi}_{s}=0.07$ и $\bar{\psi}_{p}=0.14$.

\section{Список литературы}

[1] L. Zhang, Z. Yang, T. Gong, R. Pan. J. Mater. Chem. A 8, 8813 (2020).

[2] M. Yagmurcukardes, Y. Qin, S. Ozen, M. Sayyad, F.M. Peeters, S. Tongay, H. Sahin. Appl. Phys. Rev. 7, 011311 (2020).

[3] L. Ju, M. Bie, X. Zhang, X. Chen, L. Kou. arXiv: 2009.12985.

[4] J. Zhou, Q. Wang, Q. Sun, X.S. Chen, Y. Kawazoe, P. Jena. Nano Lett. 9, 3867 (2009).

[5] S.-W. Ng, N. Noor, Z. Zheng. Asia Mater. 10, 4, 1 (2018).

[6] A. Molle, J. Goldberger, M. Houssa, Y. Xu, S.-C. Zhang, D. Akinwande. Nature Mater. 16, 163 (2017).

[7] R. Li, Y. Cheng, W. Huang. Small 1802091 (2018).

[8] L. Ju, M. Bie, J. Shang, X. Tang, L. Kou. J. Phys. Mater. 3, 022004 (2020).

[9] J. Zhou, Q. Wang, Q. Sun, P. Jena. Phys. Rev. B 81, 085442 (2010).
[10] Y. Wei, X. Tang, J. Shang, L. Ju, L. Kou. Intern. J. Smart Nano Mater. 11, 247 (2020).

[11] С.Ю. Давыдов. ФТТ 63, 413 (2021).

[12] A. Molle, J. Goldberger, M. Houssa, Y. Xu4, S.-C. Zhang, D. Akinwande. Nature Mater. 16, 163 (2017).

[13] W. Tao, N. Kong, X. Ji, Y. Zhang, A. Sharma, J. Ouyang, B. Qi, J. Wang, N. Xie, C. Kang, H. Zhang, O.C. Farokhzad, J.S. Kim. Chem. Soc. Rev. 48, 2891 (2019).

[14] У. Харрисон. Электронная структура и свойства твердых тел. Мир, М. 1983.

[15] W.A. Harrison. Phys. Rev. B 27, 3592 (1983).

[16] Физические величины. Справочник / Под ред. Е.С. Григорьева, Е.3. Мейлихова. Энергоатомиздат, М. ( 1991).

[17] Краткий справочник физико-химических величин / Под ред. К.П. Мищенко и А.А. Равделя. Химия, Л. (1974).

[18] J. Zhou, M.M. Wu, X. Zhou, Q. Sun. Appl. Phys. Lett. 95, 103108 (2009).

[19] M. Yang, R. Zhao, J. Wang, L. Zhang, Q. Xie, Z. Liu, Z. Liu. J. Appl. Phys. 113, 084313 (2013).

[20] M. Sun, Q. Ren, S. Wang, J. Yu, W. Tang. J. Phys. D 49, 445305 (2016).

[21] С.Ю. Давыдов. ФТТ 58, 779 (2016).

[22] H. Sahin, S. Cahangirov, M. Topsakal, E. Bekaroglu, E. Akturk, R.T. Senger, S. Ciraci1. Phys. Rev. B 80, 155453 (2009).

[23] С.Ю. Давыдов. ФТТ 62, 2188 (2020).

[24] С.Ю. Давыдов. ФТТ 63, 158 (2021).

[25] С.Ю. Давыдов, О.В. Посредник. ФТТ 57, 819 (2015).

[26] J. Zhou, M.M. Wu, X. Zhou, Q. Sun. Appl. Phys. Lett. 95, 103108 (2009).

[27] Ч. Киттель. Введение в физику твердого тела. Наука, М. (1978).

[28] С.Ю. Давыдов, А.А. Лебедев, О.В. Посредник. Элементарное введение в теорию наносистем. Изд-во „Лань“, СПб (2014).

[29] W.A. Harrison. Phys. Rev. B 31, 2121 (1985).

\section{Редактор Т.Н. Василевская}

